\title{
Hedging "Sudden Stops" and Emergent Recessions through International Reserves in Egypt-An Application of the Martingale Optimality Principle Approach
}

\author{
Ahmed S. Abutaleb ${ }^{1,2}$, Michael G. Papaioannou ${ }^{3 *}$ \\ ${ }^{1}$ School of Engineering, Cairo University, Giza, Egypt \\ ${ }^{2}$ MIT Lincoln Laboratory, Lexington, MA, USA \\ ${ }^{3}$ International Monetary Fund, Washington DC, USA \\ Email: ataleb@mcit.gov.eg, *michaelgpapaioannou@gmail.com
}

How to cite this paper: Abutaleb, A.S. and Papaioannou, M.G. (2021) Hedging "Sudden Stops" and Emergent Recessions through International Reserves in Egypt-An Application of the Martingale Optimality Principle Approach. Journal of Mathematical Finance, 11, 416-437.

https://doi.org/10.4236/jmf.2021.113024

Received: April 22, 2021

Accepted: July 31, 2021

Published: August 3, 2021

Copyright $\odot 2021$ by author(s) and Scientific Research Publishing Inc. This work is licensed under the Creative Commons Attribution International License (CC BY 4.0).

http://creativecommons.org/licenses/by/4.0/

\begin{abstract}
This paper addresses the role of international reserves in mitigating the adverse effects of potential "sudden stops" on economic growth. As holding of reserves implies a cost in terms of domestic consumption, we calculate the optimal level of reserves that helps hedge against such eventuality. Treating it as an optimization problem, we employ the Martingale Optimality Principle to estimate the optimal values of reserves and consumption. For Egypt, we find that optimal consumption is trending upward when the optimal level of reserves is trending downward. Using stochastic control, we obtain another approximate or suboptimal set of values for both variables. These values closely mimic Egypt's observed reserve and consumption measurements. Our findings indicate that Egypt's actual reserves during 1985-2017 exceed estimated optimal levels of reserves, even under a highly risk averse scenario, and point out to the implied consumption costs.
\end{abstract}

\section{Keywords}

Reserves, Consumption, Stochastic Control, The Martingale Optimality Principle

\section{Introduction}

Developing countries' international reserves have increased dramatically in recent years, growing by more than 60 percent since the Asian financial crisis of 1997 [1] and [2]. Some policy makers have argued that this is the best practice to 
insure against a future Balance-of-Payments crisis [3]. The implications of such self-insuring strategy are that a significant level of deadweight losses would nonetheless be incurred [4] and [5]. Despite the ongoing debate on reserve issues, there is little consensus about how to assess reserve holdings in different economies, even though this is an important aspect of any country's external stability assessment [6].

"Sudden stops" of net international capital flows to an economy can throw it to a financial crisis, often manifested by high interest rates, a sharp exchange rate depreciation, and a run on the banks, that can eventually lead to an economic crash typically evidenced by inflation increases, inability to pay for imports and service the debt, asset price corrections, declines in consumption, and ultimately a shrinkage in economic activity. Even developed economies have suffered from "sudden stops" and crashes. The recent 2010-2018 European sovereign debt crisis that engulfed Cyprus, Greece, Ireland, and Portugal is a proof of this. The situation can be even worse for emerging market (EM) economies, as the past seven decades have amply shown. Argentina is one of the most prominent EM cases, but Egypt has also experienced such adverse conditions. Funding of the government's fiscal accounts and foreign direct investments (FDI) to finance long-term projects, especially in emerging markets, is usually hard to find. In case of a crash, these economies face also intensified capital outflows, as it happened to Egypt in 2011. These serious repercussions on the economy might very well trigger a recession. In a typical "sudden stop," external funding declines by $10 \%$ or more, and persists for about a year [7].

In general, countries with poor economic performance, fixed exchange rate regimes and financial openness (high integration with financial markets) tend to be more vulnerable to "sudden stops" from global investors. To avert the negative impacts of a "sudden stop" should it occur, adequate reserves would need to be readily available to finance the country's current account deficit. Such deficit, however, would tend to narrow as a result of the reduced net capital inflows from the "sudden stop." Also, if a "sudden stop" is followed by an exchange rate depreciation, this would further diminish the current account deficit as exports would typically increase and imports decrease. It should be stressed that the availability of reserves to cover emergency balance-of-payments needs, including from "sudden stops," is over and above the required amount of reserves by monetary authorities to heighten resiliency against possible concomitant speculative attacks on the domestic currency.

In the last few years, there has been considerable effort to identify guidance on the appropriate level of reserves for less-mature market economies such as that of Egypt. The traditional adequacy rules for reserves are relatively intuitive and simple, yet at the same time they are partial and narrow in scope. Such rules include:

1) For countries with less open capital accounts, three months' coverage of imports is a typical benchmark.

2) The "Greenspan-Guidotti" rule of 100 percent cover of short-term debt is 
also a widely-used standard.

3) For countries with large banking sectors and very open capital accounts, the ratio of reserves to broad money (often proxied by M2) is typically set at 20 percent (which should be about $5 \%$ of the GDP).

4) The expanded Greenspan-Guidotti rule, consisting of short-term debt plus the current account deficit, is intended to reflect funding coverage of 12-month potential financing needs.

While there is substantial agreement on the kind of policy adjustments that reduce domestic risk, there is less consensus on what external asset management strategies should be followed to secure adequate reserves to deal with external shocks or "sudden stops." More recently, optimal reserve models were developed to integrate cost and benefit considerations. A widely used model is that of [8], where the optimal level of reserves is determined by balancing the economic cost (the potential loss in output and consumption, given the size and probability of the "sudden stop") with the opportunity cost of holding reserves, and reflecting the degree of risk aversion. An issue with this approach is that it can result in a wide range of estimated optimal reserve holdings, depending upon its calibration by the policy maker or analyst.

Recently it has been suggested that emerging economies should reduce the non-contingent part of reserves and replace it with what is known as contingent reserves [9] and [10]. Contingent reserves is a set of contracts in the stock market that move with the volatility index (VIX). The VIX index has proven to be highly correlated with the appearance of "sudden stops." The cost of buying such contracts is almost $10 \%$ or less of the cost of holding reserves. Once a "sudden stop" is detected and the VIX increases, such contracts are due and their amount is used to offset the loss in the emerging economies' current account [11].

In this paper we present exact expressions for the optimal levels of reserves and consumption. We also derive an approximate relation between consumption and reserves. For Egypt, it turns out that this suboptimal relation mimics, to a great extent, its consumption and reserves patterns. Treating reserves as assets with risk-free interest, we are able to derive an exact expression for the optimal reserves and optimal consumption. The objective function or the optimality criterion is the discounted utility of the normalized consumption. The normalization is with respect to nominal GDP. The used utility function is a constant relative risk aversion (CRRA) representation, with a parameter " $\gamma$ " reflecting the degree of risk aversion. This approach sets the optimization problem, without being concerned about the usage of reserves, while the Martingale Optimality Principle is used for the derivations [12] [13] [14].

This study is organized as follows: Section 2 presents a mathematical model for "sudden stops," outlining the behavior of the main economic agents of an emerging market economy facing a "sudden stop" and discusses the optimization problem for Egypt. Section 3 presents the results of the Martingale approach for the optimal normalized (to GDP) levels of reserves and consumption, and Section 4 discusses the results for the Egyptian economy. Section 5 provides some 
concluding remarks.

\section{2. "Sudden Stops"—The Mathematical Model}

We study a representative agent economy with a responsible government that seeks to maximize the expected present value of the society's utility from consumption $C(t)$ :

$$
E\left\{\int_{t}^{\infty} U(C(s)) \mathrm{e}^{-r(s-t)} \mathrm{d} s\right\}
$$

where $r$ is the riskless interest rate and the discount factor. The utility function $U(C(t))$ has many shapes, using in this analysis the CRRA shape as given in [15] [16]:

$$
U(C)=\frac{C^{1-\gamma}}{1-\gamma}, \quad \gamma<1, \gamma \neq 1
$$

\subsection{Emerging Market Economies and World Capital Markets}

This section presents an analytical framework of behavior of domestic absorption, output, and reserves in emerging market economies (EMs) experiencing sudden stops in their capital flows.

Let $Y(t)$ represent the country's income (GDP) in its pre-development phase, and assume that it follows the Geometric Brownian motion model. The stochastic differential equation (SDE) of $Y(t)$ is given by:

$$
\mathrm{d} Y(t)=\mu_{\mathrm{Y}} Y(t) \mathrm{d} t+\sigma_{Y} Y(t) \mathrm{d} B(t)
$$

where $\mu_{Y}$ is the growth rate of GDP, $\sigma_{Y}$ is the growth rate volatility, and $B(t)$ is the Brownian motion or the Wiener process.

$Y(t) \mathrm{SDE}$ has the solution:

$$
\ln \left(\frac{Y(t)}{Y(0)}\right)=\left(\mu_{Y}-\frac{\sigma_{Y}^{2}}{2}\right) t+\sigma_{Y} B(t)
$$

For Egypt, and expressing all variables in current US Dollar terms, we get:

$\left(\mu_{Y}-\frac{\sigma_{Y}^{2}}{2}\right)=0.07724$. Using the maximum likelihood method, we get:

$\mu_{Y}=0.08, \sigma_{Y}^{2}=0.0062$.

In its developing stage, a country would like to borrow against its post development income. Potential financing agents include: 1) broad world capital market creditors (WCM), and 2) specialized in EMs creditors (specialists). Unlike specialists, WCM have limited understanding of emerging markets and hence do not accept contracts related to EMs. At this stage, the country can also accumulate international assets $X(t)$. Both assets and liabilities pay a return $\mathrm{r}$ per unit of time.

\section{2. "Sudden Stops" and Specialists}

Specialists, as investors familiar with EMs at large, are willing to invest in many areas where WCM investors do not feel comfortable (e.g., due to lack of exper- 
tise) or are not permitted (e.g., due to risk management considerations) to invest. In practice, they deal with equity investments, FDI, the riskiest tranches of GDPindexed bonds, and toxic-assets in general. Thus, during non-sudden stop times "NSS" or normal times, the maximum flow of resources received is " $\bar{f} Y(t)$ ":

$$
\max f^{N S S}(t)=\bar{f}
$$

During sudden stops, the maximum flow of resources received from special-

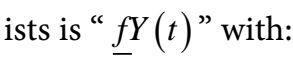

$$
\underline{f}<\bar{f}
$$

Thus,

$$
f^{S S}(t)=\underline{f}
$$

We define now $A(t)$ as the sum of income and contingent flows from specialists:

$$
A(t)=\left(\theta^{N S S} 1\{N S S\}+\theta^{S S} 1\{S S\}\right) Y(t)
$$

where

$$
\begin{gathered}
\theta^{\text {NSS }}(t) \leq(1+\bar{f}) \\
\theta^{S S}(t) \leq(1+\underline{f}) \\
1\{N S S\}=\left\{\begin{array}{lc}
1 & \text { Country in normal times } \\
0 & \text { elsewhere }
\end{array}\right. \\
1\{S S\}=\left\{\begin{array}{lc}
1 & \text { Country in sudden stop times } \\
0 & \text { elsewhere }
\end{array}\right.
\end{gathered}
$$

Note that $\theta^{S S}<\theta^{\text {NSS }}$

The net asset accumulation or reserves $X(t)$ is now described by:

$$
\begin{gathered}
\mathrm{d} X(t)=[r X(t)-C(t)+A(t)] \mathrm{d} t \\
A(t)=Y(t)+\text { "net foreign accumulation" }
\end{gathered}
$$

The change in reserves is due to (1) interest on reserves at $r(\%)$, and (2) the difference between the "GDP + net foreign accumulation", $A(t)$, and the consumption $C(t)$. Using the conventional national income accounting definitions, the consumption, $C(t)$, is defined as the sum of government consumption, household consumption, plus private investment, while $C(t)$ equals to GDP + Current Account balance. Note that Equation (2.12) shows a negative relationship between international reserves, $X(t)$, and the domestic absorption or the consumption $C(t)$.

\subsection{An Illustrative Country Case-Egypt}

We define the normalized variables $c(t)=C(t) /(\psi Y(t))$ and $x(t)=X(t) /(\psi Y(t))$, where $\psi$ is a constant value that could be 1 or assume 
other values. It can be noted that the relation between $c(t)$ and $x(t)$ follows what is known as a "prey-predator" equation [17]. As $c(t)$ increases, $x(t)$ decreases and then $c(t)$ decreases and $x(t)$ increases in a cyclical behavior. We have calculated this cycle, using Fourier transform, to be around 7 years. This is shown in Figure 1, where we present the data for 1985-2017.

\subsection{The Optimization Problem}

To study the impact of a "sudden stop" (temporary shock) on an economy, we will first study the case that the economy is normal, i.e., does not face "sudden stops" (NSS).

We define:

$$
\begin{aligned}
V(X(0), Y(0)) & =\max _{C(s)} E\left\{\int_{0}^{\tau^{s S}} \mathrm{e}^{-r s} U(C(s)) \mathrm{d} s+\varphi\left(X\left(\tau^{s S}\right), Y\left(\tau^{S S}\right)\right)\right\} \\
& =\text { value function in the normal state }
\end{aligned}
$$

$\varphi\left(X\left(\tau^{S S}\right), Y\left(\tau^{S S}\right)\right)=$ Utility of the desired value of reserves at the onset of $S S$

$\tau^{S S}=$ time to sudden stop, which is random

$$
U(C)=\frac{C^{1-\gamma}}{1-\gamma}, \gamma>0, \gamma \neq 1
$$

Transition from normal times to sudden stops occur with a constant hazard rate $\lambda$ at the random time $\tau^{S S}$. The constraints and the system dynamics are (see Equation (2.3)):

$$
A(t)=\theta^{N S S} Y(t)
$$

$\theta^{\text {NSS }}$ for developing countries is around $1.2[10]$.

It turns out that $\theta^{\text {NSS }}$ for Egypt is around 1, but is changing over time (it is taken to be $A(t) / Y(t))$.

$$
\begin{gathered}
\mathrm{d} X(t)=\left[r X(t)-C(t)+\theta^{N S S} Y(t)\right] \mathrm{d} t \\
X(t) \geq 0
\end{gathered}
$$

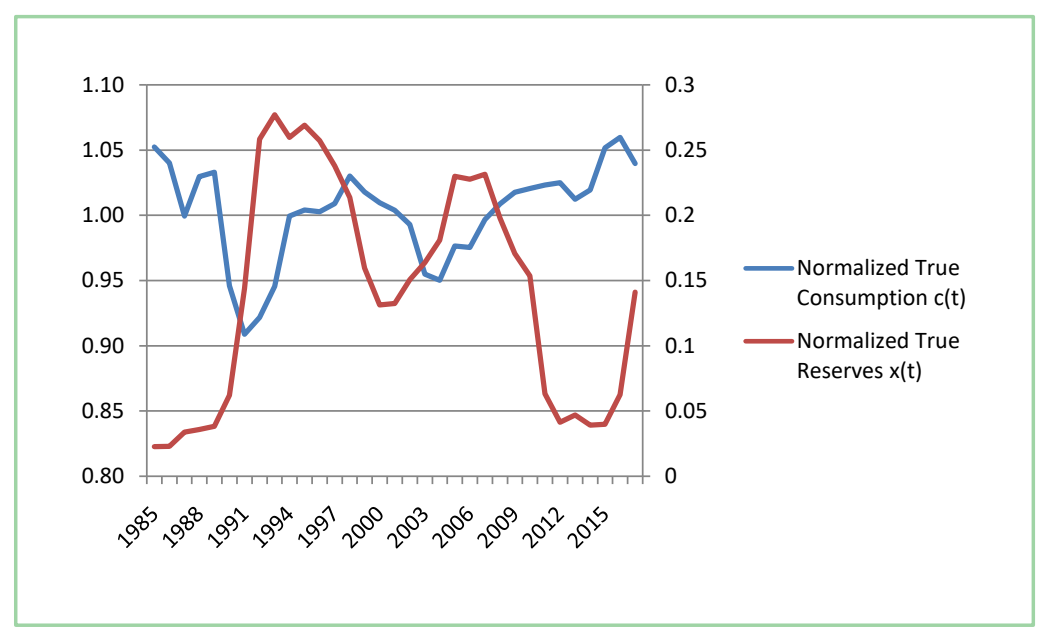

Figure 1. Normalized true consumption and reserves for Egypt. 
In vector format, Equations (2.3), (2.11) and (2.12) are written compactly as:

$$
\left[\begin{array}{l}
\mathrm{d} X(t) \\
\mathrm{d} Y(t)
\end{array}\right]=\left\{\left[\begin{array}{cc}
r & \theta^{N S S} \\
0 & \mu_{Y}
\end{array}\right]\left[\begin{array}{l}
X(t) \\
Y(t)
\end{array}\right]+\left[\begin{array}{c}
-C(t) \\
0
\end{array}\right]\right\} \mathrm{d} t+\left[\begin{array}{c}
0 \\
\sigma_{Y} Y(t)
\end{array}\right] \mathrm{d} B(t)
$$

Then, the country is faced with the decision of how much to consume $C(t)$ in order to maximize the utility function $U(C)$ and at the same time to end with a desired level of reserves $X\left(\tau^{s S}\right)$, with a utility function $\varphi\left(X\left(\tau^{S S}\right), Y\left(\tau^{S S}\right)\right)$. The final value of reserves $X\left(\tau^{S S}\right)$ could be defined by the decision maker or optimally estimated. The reserves, $X(t)$, play the role of providing the country with adequate financial resources to cope with a reduction or complete stop of capital inflows during a "sudden stop." Accumulating reserves, however, is costly and deprives the economy from precious resources that could be used for GDP growth and development.

The optimization problem entails the finding of the optimal consumption path and at the same time satisfying the level of the desired level of reserves at the end time of a "sudden stop" $\tau^{S S}$. After normalization with respect to $\psi Y(t)$, we get (see Appendix A):

$$
\mathrm{d} x(t)=\left[x(t)\left(r-\mu_{Y}+\sigma_{Y}^{2}\right)+\left(\left(\frac{\theta^{N S S}}{\psi}\right)-c(t)\right)\right] \mathrm{d} t-x(t) \sigma_{Y} \mathrm{~d} B(t)
$$

where $c(t)=C(t) / \psi Y(t)$ and $x(t)=X(t) / \psi Y(t)$.

An approximate solution is obtained by assuming time-invariance of the objective function. Then, the optimal normalized consumption $c(x)$ as function of the normalized $x(t)$ reserves is derived as (see Appendix A):

$$
c(x)=K_{1}+K_{2} \frac{x^{\frac{2}{\sigma_{Y} \gamma}}}{1-x^{\frac{2}{\sigma_{Y} \gamma}}}
$$

where $K_{1}$ and $K_{2}$ are constants to be determined from the boundary conditions or by minimizing the sum of squared error between the observed $c(t)$ and the estimated $c$ of Equation (A.8) and using the observed $x(t)$. The relationship between $c(t)$ and $x(t)$ of Equation (A.8) is close to the "prey-predator" model [17]. This implies that the approximate optimal solution reflects what we observe in reality.

An approximate SDE of the normalized reserves becomes see Equation (A.9).

From Equations (2.12) and (A.8), we may observe that we have two mechanisms at play: one generating a negative correlation between international reserves and absorption (the SDE of Equation (2.12)) and another generating a positive correlation between absorption and reserves (the optimization Equation (A.8). This constitutes what is known as a feedback mechanism, which ensures that the economy is stable.

\section{Optimal Outcomes}

\subsection{Optimal Normalized Reserves and Optimal Normalized Consumption-The Martingale Approach}

In this section, we define the objective function as (see Appendix A, Equation (A.11)). 
Here we maximize the discounted utility function of $c(s), \mathrm{e}^{-\rho s} U(c(s))$, and minimize the utility function of the final value of the normalized reserves $U_{x}\left(x\left(\tau^{S S}\right)\right)$. Through the maximization (see Appendix A), we are able to determine the exact optimal values of $c(t)$ and the corresponding optimal reserves $x(t)$. Unlike with other methods, we do not give a prescribed desired value for $x\left(\tau^{S S}\right)$. The derived equation for the optimal values sees Equation (A.23).

Equation (A.23) shows that the optimal normalized consumption $c(t)$ follows a Geometric Brownian motion with a linear trend coefficient. For the estimated values of Egypt, the trend is $\left(\frac{1}{\gamma}\right)\left[r+\frac{(1+1 / \gamma)}{2}\left(\frac{\left(\mu_{Y}-\sigma_{Y}^{2}\right)}{\sigma_{Y}}\right)^{2}-\rho\right]>0$. The value of $\gamma$ reflects the level of conservatism-as $\gamma$ increases, we get more conservative results.

The corresponding optimal values of the normalized reserves $x(t)$ are obtained by the substitution of Equation (A.23) into the following:

$$
\mathrm{d} x(t)=\left[x(t)\left(r-\mu_{Y}+\sigma_{Y}^{2}\right)+\left(\left(\frac{\theta^{N S S}}{\psi}\right)-c(t)\right)\right] \mathrm{d} t-x(t) \sigma_{Y} \mathrm{~d} B(t)
$$

Note that $\left(\left(\frac{\theta^{\text {NSS }}}{\psi}\right)-c(t)\right)>0$, with this being a constraint on $c(t)$. The value of $\left(\frac{\theta^{N S S}}{\psi}\right)$ is around 1.0 in most of the analysis. Remember that $\left(\frac{\theta^{N S S}}{\psi}\right)=A(t) / Y(t)$.

\subsection{Portfolio Decision}

In 2017, Egypt's reserves stood at around 14 percent of GDP. We set as an objective the gradual reduction of this amount to a fixed level over a few years. At the same time, Egypt should possess assets at an amount that attends to the central bank's liquidity operations and is enough to ensure the coverage of several months of imports in case that a "sudden stop" (SS) occurs. The basic premise is that existing reserves exceed required reserves for these functions. In essence, starting with a high value of reserves, $X(t)$, we need to reduce this quantity gradually to a final fixed value $X_{C}$. At the time of an SS, we assume that there will be a certain payoff obtained from an external sovereign fund. The sum of the two, i.e., $X_{C}$ and external payoff, should be close to a target value that might be equal to, for example, 10 months of imports. This policy will reduce the needed cash reserves from the current high levels to the value $X_{C}$, with the reduction in reserves freeing resources to be infused in the economy. This will act as a stimulus to the domestic economy, leading to higher GDP growth rates.

\section{Discussion of Results for the Egyptian Economy}

In this section we present scenarios that result in optimal reserves, by 2021, of $5 \%$ and $10 \%$ of the GDP, along with the corresponding optimal consumption. (Oth- 
er scenarios could have been designed using the same approach.) Then, we compare the obtained optimal levels of consumption to the actual measured levels during the period 1985-2017. During this period, we use

$\left(\frac{\theta^{\text {NSS }}}{\psi}\right)=A(t) / Y(t)$, with $\psi=1$. Also, we present forecasts until 2021. In the forecast period, 2018-2021, we set $\left(\frac{\theta^{\text {NSS }}}{\psi}\right)$ of Equation (A.3) to be exactly 1. All figures have the average values of $c(t)$ and $x(t)$.

We find that the optimal consumption is in the same order of magnitude as the actual one in most of the period under study (see Figure 2).

Note that the optimal normalized consumption, $c(t)$, is around 1 . This is consistent with the findings of [8] and [10].

We also find that the optimal levels of the normalized reserves are lower than the actual ones for most of the time (see Figure 3).

The above results in Figure 2 and Figure 3 were obtained by setting $\gamma=15$. For a more conservative approach, we present the same results with $\gamma=25$ (see Figures 4-6).

We note that even in the conservative approach, where the monetary authorities' risk aversion is high, $\gamma=25$, as compared to a more risk taking approach, $\gamma=15$, the levels of the estimated optimal normalized reserves are lower than the true (actual, observed) reserves for the period 1985-2017 (see Figure 7). Based on our methodology, these results indicate that monetary authorities had been excessively prudent in setting international reserve targets during the studied period. However, the observed reserve levels may be justified if the authorities presumed that required reserves had to be higher than the common tenmonth import coverage that we assumed in our analysis. The assessment of the adequacy of reserves at times tends not to follow conventional norms, but rather to be subjective due to perceived risks and circumstantial due to domestic and external economic and political conditions [2]. In such cases, monetary authorities would need to be transparent by disclosing the reasons for setting higher-than-

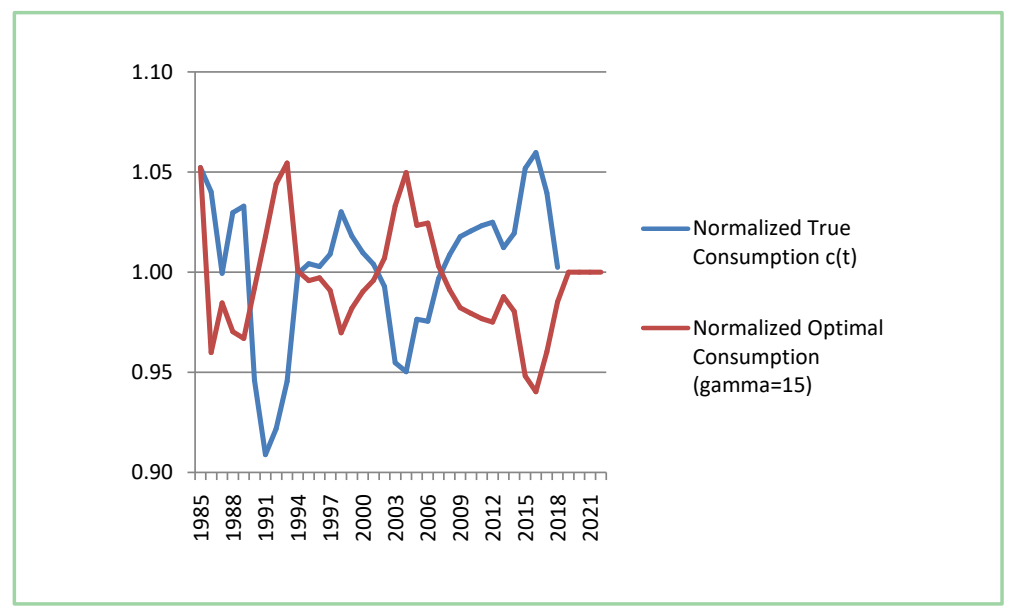

Figure 2. True and optimal normalized consumption (gamma $=15)$. 


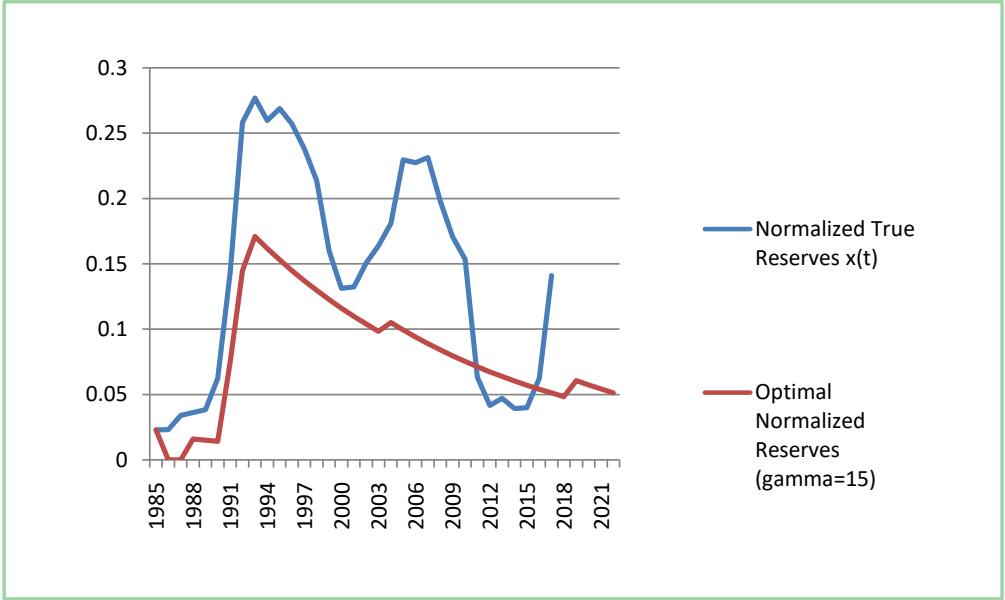

Figure 3. True and optimal normalized reserves (gamma $=15)$.

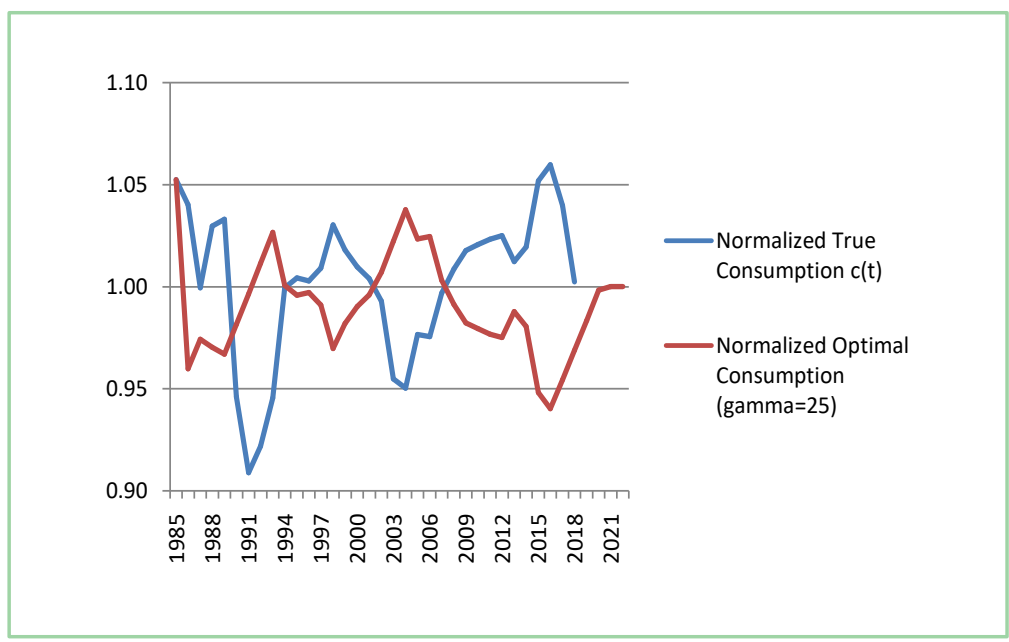

Figure 4. True and optimal normalized consumption (gamma $=25)$.

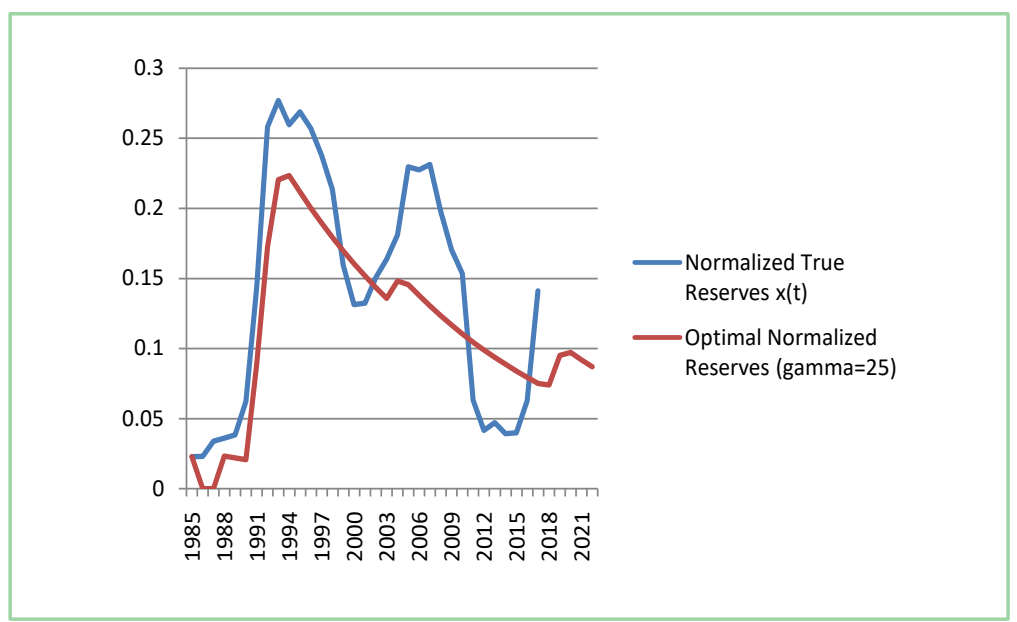

Figure 5. True and optimal normalized reserves (gamma $=25)$.

normal targets for reserves and thus justify why the economy has to suffer the costs of holding additional reserves. 


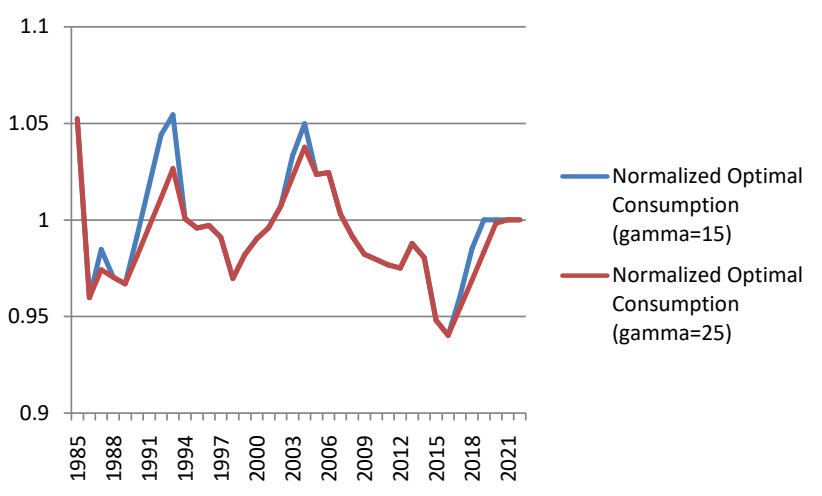

Figure 6. Comparison between conservative (gamma $=25$ ) and risky (gam$\mathrm{ma}=15$ ) optimal consumption.

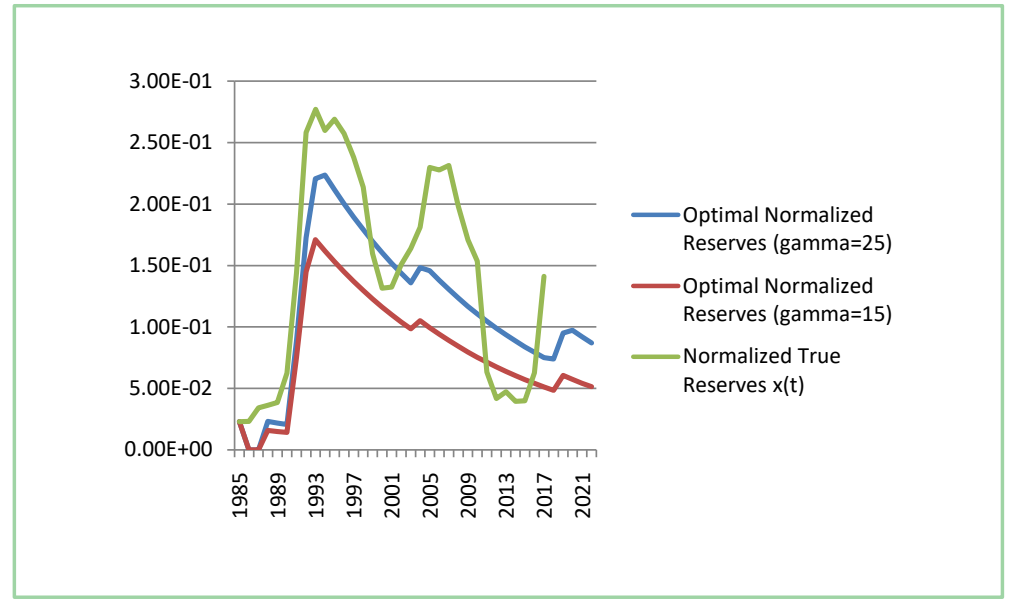

Figure 7. Comparison between conservative (gamma $=25)$ and risky (gam$\mathrm{ma}=15$ ) optimal reserves.

\section{Concluding Remarks}

We have developed a mathematical model that describes the behavior of international reserves and the corresponding consumption, when reserves are used to hedge a country's exposure to potential capital market sudden stops and emergent recessions. The model allows the decision maker to change the different economic parameters and track their effects on the optimal values of reserves and consumption. Based on minimal assumptions, the derived optimal levels indicate that, for Egypt, the monetary policy maker had opted for higher reserves than their optimal values estimated even for the higher risk aversion approach. However, by taking a less aggressive approach, reserves would have been freed and propelled into the economy, leading to an increase in Egypt's GDP rate of growth. Nevertheless, we recognize that special circumstances may have necessitated the tilting of the inherent trade-off towards higher levels of reserves to secure economic stability and consequently lower economic activity and consumption. Ideally, such decisions by monetary authorities should be commu- 
nicated clearly to raise public's awareness to needed sacrifices and thus avoid social discontent.

\section{Conflicts of Interest}

The authors declare no conflicts of interest regarding the publication of this paper.

\section{References}

[1] Mendoza, R. (2004) International Reserve-Holding in the Developing World: Self Insurance in a Crisis-Prone Era. Emerging Markets Review, 5, 61-82. https://doi.org/10.1016/j.ememar.2003.12.003

[2] Gapen, M. and Papaioannou, M. (2008) International Reserves Accumulation: Some Lessons from Asia. In: Kurihara, Y., Takaya, S., Harui, H. and Kamae, H., Eds., Information Technology and Economic Development, Chapter VII, IGI Global, Hershey, 88-104. https://doi.org/10.4018/978-1-59904-579-5.ch007

[3] European Central Bank (ECB) (2006) The Accumulation of Foreign Reserves. International Relations Committee Task Force, Occasional Paper Series, No. 43, European Central Bank, Frankfurt am Main.

[4] Calvo, G. (1998) Capital Flows and Capital Market Crises: The Simple Economics of Sudden Stops. Journal of Applied Economics, 1, 35-54. https://doi.org/10.1080/15140326.1998.12040516

[5] Calvo, G., Izquierdo, A. and Mejia, L. (2004) On the Empirics of Sudden Stops: The Relevance of Balance-Sheet Effects. NBER Working Paper 10520. https://doi.org/10.3386/w10520

[6] IMF (2015) Assessing Reserve Adequacy-Specific Proposals. IMF, Washington DC.

[7] Cecchetti, S. and Schoenholtz, K. (2018) Sudden Stops: A Primer on Balance-onPayments Crises. VOX EU, 9 July.

[8] Jeanne, O. and Ranciere, R. (2006) The Optimal Level of International Reserves for Emerging Market Countries: Formulas and Applications. IMF Working Paper WP/ 06/229. https://doi.org/10.5089/9781451864892.001

[9] Caballero, R. and Panageas, S. (2003) Hedging Sudden Stops and Precautionary Recessions: A Quantitative Framework. NBER Working Paper 9778.

https://doi.org/10.3386/w9778

[10] Caballero, R. and Panageas, S. (2004) A Quantitative Model of Sudden Stops and External Liquidity Management. NBER Working Paper 11293.

http://www.nber.org/papers/w11293

https://doi.org/10.3386/w11293

[11] Abutaleb, A. and Gaber, M. (2012) Optimal Levels of Reserves and Hedging Sudden Stops Recessions for Egypt: A Stochastic Control Approach. Journal of Economic Cooperation and Developments, 33, 1-58.

[12] Abutaleb, A. and Papaioannou, M. (2021) Introduction to the Stochastic Calculus and the Malliavin Calculus with Applications to Engineering and Finance. IDEA Group Publications, Hershey.

[13] Abutaleb, A. (2013) The Estimation of the Instantaneous Amplitudes of Sum of Sinusoids with Unknown Frequencies and Phases: The Martingale Approach. Signal Processing, 93, 811-817. https://doi.org/10.1016/j.sigpro.2012.10.003

[14] Mangel, M. (1985) Decision and Control in Uncertain Resource Systems. Academic 
Press, Cambridge.

[15] Munk, C. (2012) Dynamic Asset Allocation. https://sites.google.com/view/clausmunk/home

[16] Zhang, A. (2007) Life Insurance Applications of the Martingale Method. Ph.D. Dissertation, Department of Financial Mathematics, Fraunhofer Institute, Munich.

[17] Rao, F. (2013) Dynamical Analysis of a Stochastic Predator-Prey Model with an Allee Effect. Abstract and Applied Analysis, 2013, Article ID: 340980.

https://doi.org/10.1155/2013/340980 


\section{Appendix A. Optimal Consumption and Optimal Reserves}

In this appendix, we derive the consumption $C(t)$ that will optimize the expected value of the utility function subject to the constraints of the system dynamics. Instead of working with the reserves $X(t)$, we will, for mathematical convenience purposes, use the normalized reserves, $x(t)$, and the normalized consumption, $c(t)$, defined as:

$$
\begin{aligned}
& x(t)=\frac{X(t)}{\psi Y(t)} \\
& c(t)=\frac{C(t)}{\psi Y(t)}
\end{aligned}
$$

GDP, $Y(t)$, and reserves, $X(t)$, evolve according to the SDE's:

$$
\begin{gathered}
\mathrm{d} Y(t)=\mu_{Y} Y(t) \mathrm{d} t+\sigma_{Y} Y(t) \mathrm{d} B(t), \quad 0<r<\mu_{Y} \\
\mathrm{~d} X(t)=\left[r X(t)-C(t)+\theta^{N S S} Y(t)\right] \mathrm{d} t \\
X(t) \geq 0
\end{gathered}
$$

An SDE for $x(t)$ :

We will derive an SDE for $x(t)=\frac{X(t)}{\psi Y(t)}$, for a non-sudden stop, using Ito's lemma:

$$
\mathrm{d} x=\frac{\partial X}{\partial t} \mathrm{~d} t+\frac{\partial x}{\partial X} \mathrm{~d} X+\frac{\partial X}{\partial Y} \mathrm{~d} Y+\frac{1}{2} \frac{\partial^{2} X}{\partial X^{2}}(\mathrm{~d} X)^{2}+\frac{\partial^{2} X}{\partial X \partial Y} \mathrm{~d} X \mathrm{~d} Y+\frac{1}{2} \frac{\partial^{2} X}{\partial Y^{2}}(\mathrm{~d} Y)^{2} \text { (A.2) }
$$

Since $x(t)=\frac{X(t)}{\psi Y(t)}$

then, $\frac{\partial x}{\partial t}=0$

$$
\begin{gathered}
\frac{\partial x}{\partial X}=\frac{1}{\psi Y} \\
\frac{\partial X}{\partial Y}=\frac{-\psi X}{(\psi Y)^{2}}=\frac{-X}{\psi Y^{2}} \\
\frac{\partial^{2} X}{\partial X^{2}}=0 \\
\frac{\partial^{2} x}{\partial X \partial Y}=\frac{-1}{\psi Y^{2}} \\
\frac{\partial^{2} X}{\partial Y^{2}}=\frac{2 X}{\psi Y^{3}} \\
(\mathrm{~d} Y)^{2}=\sigma_{Y}^{2} Y^{2} \mathrm{~d} t \\
(\mathrm{~d} X)^{2}=0 \\
\mathrm{~d} X \mathrm{~d} Y=0
\end{gathered}
$$


Substituting for the partial derivatives in Equation (A.2), we get:

$$
\mathrm{d} x=\frac{1}{\psi Y} \mathrm{~d} X-\frac{X}{\psi Y^{2}} \mathrm{~d} Y-\frac{1}{\psi Y^{2}} \mathrm{~d} X \mathrm{~d} Y+\frac{X}{\psi Y^{3}}(\mathrm{~d} Y)^{2}
$$

Substituting for the complete derivatives, we get:

$$
\begin{aligned}
\mathrm{d} x= & \frac{1}{\psi Y}\left[r X(t)-C(t)+\theta^{N S S} Y(t)\right] \mathrm{d} t-\frac{X}{\psi Y^{2}}\left[\mu_{Y} Y(t) \mathrm{d} t+\sigma_{Y} Y(t) \mathrm{d} B(t)\right] \\
& +0+\frac{X}{\psi Y^{3}} \sigma_{Y}^{2} Y^{2} \mathrm{~d} t
\end{aligned}
$$

which is reduced to:

$$
\mathrm{d} x=\frac{X}{\psi Y(t)}\left[r-\frac{C(t)}{X(t)}+\frac{\theta^{N S S} Y(t)}{X(t)}\right] \mathrm{d} t-\frac{X(t)}{\psi Y(t)}\left[\mu_{\mathrm{Y}} \mathrm{d} t+\sigma_{Y} \mathrm{~d} B(t)\right]+\frac{X(t)}{\psi Y(t)} \sigma_{Y}^{2} \mathrm{~d} t
$$

By rearranging terms, we get:

$$
\begin{aligned}
\mathrm{d} x & =x(t)\left[r-\frac{C(t)}{X(t)}+\frac{\theta^{N S S} Y(t)}{X(t)}\right] \mathrm{d} t-x(t)\left[\mu_{Y} \mathrm{~d} t+\sigma_{Y} \mathrm{~d} B(t)\right]+x(t) \sigma_{Y}^{2} \mathrm{~d} t \\
& =x(t)\left[\left(r-\mu_{Y}+\sigma_{Y}^{2}\right)-\frac{C(t)}{X(t)}+\frac{\theta^{N S S} \psi Y(t)}{\psi X(t)}\right] \mathrm{d} t-x(t) \sigma_{Y} \mathrm{~d} B(t) \\
& =x(t)\left[\left(r-\mu_{Y}+\sigma_{Y}^{2}\right)-\frac{c(t)}{x(t)}+\left(\frac{\theta^{N S S}}{\psi}\right) \frac{1}{x(t)}\right] \mathrm{d} t-x(t) \sigma_{Y} \mathrm{~d} B(t)
\end{aligned}
$$

and

$$
\mathrm{d} x(t)=\left[\left(\frac{\theta^{N S S}}{\psi}\right)+x(t)\left(r-\mu_{Y}+\sigma_{Y}^{2}\right)-c(t)\right] \mathrm{d} t-x(t) \sigma_{Y} \mathrm{~d} B(t)
$$

Equation (A.3) is the desired SDE that describes the evolution of normalized reserves $x(t)$.

\section{Exact Solution for the Normalized Variables:}

Because all the components are explicitly independent of time and the diffusion is independent of the control, we will be able to derive an exact ODE for the control, $c(x)$ [12] and [14]. The SDE describing the normalized reserves is given by:

$$
\mathrm{d} x(t)=x(t)\left[\left(r-\mu_{Y}+\sigma_{Y}^{2}\right)-\frac{c(t)}{x(t)}+\frac{1}{x(t)}\right] \mathrm{d} t-x(t) \sigma_{Y} \mathrm{~d} B(t)
$$

where we have set $\left(\frac{\theta^{\text {NSS }}}{\psi}\right)=1$

The normalized utility function is given by [15]:

$$
U(c)=\frac{c^{1-\gamma}}{1-\gamma}, \gamma>0, \gamma \neq 1
$$

The objective function is given by:

$$
V(x(t))=\max _{c(s)} E\left\{\int_{t}^{\tau^{s S}} U(c(s)) \mathrm{d} s+\varphi_{1}\left(x\left(\tau^{s S}\right)\right)\right\}
$$


where all variables involved are normalized and, for simplification and to find a closed form solution, the discount factor $\mathrm{e}^{-r(s-t)}$ is eliminated.

The optimal normalized consumption $c(t)$ satisfies the ordinary differential equation (ODE) [12]:

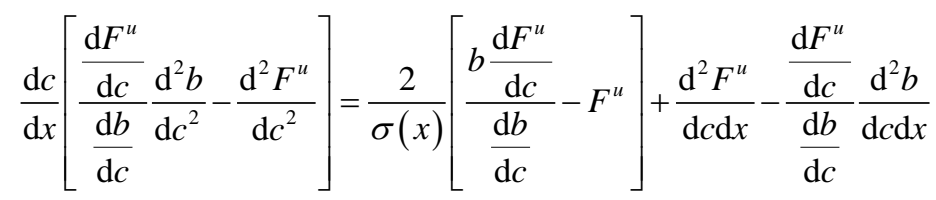

where $u(x)=c(x)$

$$
\begin{gathered}
F^{u}(c)=\frac{c^{1-\gamma}}{1-\gamma}, \gamma>0, \gamma \neq 1 \\
b(x, c)=x\left[\left(r-\mu_{Y}+\sigma_{Y}^{2}\right)-\frac{c}{x}+\frac{1}{x}\right] \\
\sigma(x)=-x \sigma_{Y}
\end{gathered}
$$

We need the following derivatives:

$$
\begin{gathered}
\frac{\mathrm{d} F^{u}(c)}{\mathrm{d} c}=c^{-\gamma} \\
\frac{\mathrm{d}^{2} F^{u}(c)}{\mathrm{d} c^{2}}=-\gamma c^{-\gamma-1} \\
\frac{\mathrm{d}^{2} F^{u}(c)}{\mathrm{d} c \mathrm{~d} x}=0 \\
\frac{\mathrm{d} b(x, c)}{\mathrm{d} c}=-1 \\
\frac{\mathrm{d}^{2} b(x, c)}{\mathrm{d} c^{2}}=0 \\
\frac{\mathrm{d}^{2} b(x, c)}{\mathrm{d} c \mathrm{~d} x}=0
\end{gathered}
$$

Substituting the different elements in Equation (A.6), we get:

$$
\frac{\mathrm{d} c}{\mathrm{~d} x}\left[\gamma c^{-\gamma-1}\right]=-\frac{2}{x \sigma_{Y}}\left[-x\left[\left(r-\mu_{Y}+\sigma_{Y}^{2}\right)-\frac{c}{x}+\frac{1}{x}\right] c^{-\gamma}-\frac{c^{1-\gamma}}{1-\gamma}\right]
$$

Dividing both sides by $c^{-\gamma}$ and rearrange, we get:

$$
\begin{aligned}
& \frac{\mathrm{d} c}{\mathrm{~d} x}=\frac{2 c}{x \sigma_{Y} \gamma}\left[x\left[\left(r-\mu_{Y}+\sigma_{Y}^{2}\right)-\frac{c}{x}+\frac{1}{x}\right]+\frac{c}{1-\gamma}\right] \\
& =\frac{2 c}{\sigma_{Y} \gamma}\left[\left(r-\mu_{Y}+\sigma_{Y}^{2}\right)-\frac{c}{x}+\frac{1}{x}\right]+\frac{2 c^{2}}{x \sigma_{Y} \gamma(1-\gamma)} \\
& =\frac{2 c}{\sigma_{Y} \gamma}\left[\left(r-\mu_{Y}+\sigma_{Y}^{2}\right)-\frac{c}{x}+\frac{1}{x}+\frac{c}{x(1-\gamma)}\right] \\
& =\frac{2 c}{\sigma_{Y} \gamma}\left[\left(r-\mu_{Y}+\sigma_{Y}^{2}\right)+\frac{-c(1-\gamma)+(1-\gamma)+c}{x(1-\gamma)}\right]
\end{aligned}
$$




$$
\begin{aligned}
& =\frac{2 c}{\sigma_{Y} \gamma}\left[\left(r-\mu_{Y}+\sigma_{Y}^{2}\right)+\frac{\gamma c+(1-\gamma)}{x(1-\gamma)}\right] \\
& =\frac{2 c}{\sigma_{Y} \gamma}\left[\left(r-\mu_{Y}+\sigma_{Y}^{2}\right)+\frac{\gamma c+(\gamma-1)}{x(\gamma-1)}\right] \\
& =\frac{2 c}{\sigma_{Y} \gamma}\left[\left(r-\mu_{Y}+\sigma_{Y}^{2}\right)+\frac{\gamma c}{x(\gamma-1)}+\frac{(\gamma-1)}{x(\gamma-1)}\right] \\
& \frac{\mathrm{d} c}{\mathrm{~d} x}=\frac{2 c}{\sigma_{Y} \gamma}\left[\left(r-\mu_{Y}+\sigma_{Y}^{2}\right)+\left(\frac{c}{x}\right) \frac{\gamma}{(\gamma-1)}+\frac{1}{x}\right]
\end{aligned}
$$

This is the desired ODE for the optimal normalized consumption $c(x)$, as function of the normalized reserves $x$.

\section{Some approximations:}

We know that $c$ is in the order of 1 , while $x$ is in the order 0.2 , and assuming that $\gamma \gg 1$ and $\left(r-\mu_{Y}+\sigma_{Y}^{2}\right) \ll\left(\frac{1}{x}\right)$, then

$$
\frac{\mathrm{d} c}{\mathrm{~d} x} \approx \frac{2 c}{\sigma_{Y} \gamma}\left(\frac{c+1}{x}\right)
$$

Separating variables, we get:

$$
\frac{\mathrm{d} c}{c(c+1)} \approx \frac{2}{\sigma_{Y} \gamma}\left(\frac{\mathrm{d} x}{x}\right)
$$

which has the solution:

$$
\int \frac{\mathrm{d} c}{c(c+1)}=\ln c-\ln (c+1)+\ln K=\ln \frac{K c}{c+1} \approx \frac{2}{\sigma_{Y} \gamma} \ln x
$$

where $K$ is the constant of integration, determined from the boundary conditions.

Thus,

$$
\frac{K c}{c+1} \approx x^{\frac{2}{\sigma_{Y} \gamma}}
$$

which yields $c=\frac{x^{\frac{2}{\sigma_{Y \gamma}}}}{K-x^{\frac{2}{\sigma_{Y \gamma}}}}$,

A better approximate formula is:

$$
c=K_{1}+K_{2} \frac{x^{\frac{2}{\sigma_{Y} \gamma}}}{1-x^{\frac{2}{\sigma_{Y} \gamma}}}
$$

Substituting in the normalized reserves equation, we get:

$$
\begin{aligned}
\mathrm{d} x(t)= & x(t)\left[\left(r-\mu_{Y}+\sigma_{Y}^{2}\right)-\frac{1}{x(t)}\left(K_{1}+K_{2} \frac{x^{\frac{2}{\sigma_{Y \gamma}}}}{1-x^{\frac{2}{\sigma_{Y} \gamma}}}\right)+\left(\frac{\theta^{N S S}}{\psi}\right) \frac{1}{x(t)}\right] \mathrm{d} t \\
& -x(t) \sigma_{Y} \mathrm{~d} B(t)
\end{aligned}
$$


In the simulation, we define the desired final value of $x\left(\tau^{s s}\right)$ and find the corresponding optimal values for $K_{1}$ and $K_{2}$ that satisfy, in the minimum squared error sense, this final value. We also estimate the optimal normalized reserves through the SDE of $x(t)$, and the consumption that satisfies the equation $c(t)=K_{1}+K_{2} \frac{x^{\frac{2}{\sigma_{Y} \gamma}}}{1-x^{\frac{2}{\sigma_{Y} \gamma}}}$.

\section{The Martingale Approach with $c(t)$ :}

In this subsection we use the Martingale Optimality Principle [12] [13] and [16] to find the optimal values of the normalized consumption and consequently the optimal levels of the normalized reserves.

The system dynamics are:

$$
\begin{aligned}
\mathrm{d} x(t) & =x(t)\left[\left(r-\mu_{Y}+\sigma_{Y}^{2}\right)-\frac{c(t)}{x(t)}+\left(\frac{\theta^{N S S}}{\psi}\right) \frac{1}{x(t)}\right] \mathrm{d} t-x(t) \sigma_{Y} \mathrm{~d} B(t) \\
& =\left[x(t)\left(r-\mu_{Y}+\sigma_{Y}^{2}\right)+\left(\left(\frac{\theta^{N S S}}{\psi}\right)-c(t)\right)\right] \mathrm{d} t-x(t) \sigma_{Y} \mathrm{~d} B(t)
\end{aligned}
$$

The normalized utility function is given as:

$$
U(c(t))=\frac{c^{1-\gamma}}{1-\gamma}, \gamma>0, \gamma \neq 1
$$

In this analysis, we define the objective function as:

$$
V(x(0))=\max _{c(s), x\left(\tau^{S S}\right)} E\left\{\int_{0}^{s S} \mathrm{e}^{-\rho s} U(c(s)) \mathrm{d} s-U_{x}\left(x\left(\tau^{s S}\right)\right)\right\}
$$

Notice that $U_{x}\left(x\left(\tau^{S S}\right)\right)$ is not defined, because it will be of no use for the derivation of the SDE of the optimal $c(t)$.

In the martingale approach, we need to find the process $H(t)$ such that $H(t) x(t)-H(t) \int\left(\left(\frac{\theta^{\text {NSS }}}{\psi}\right)-c(s)\right) \mathrm{d} s$ is a martingale. Assume that $H(t)$ has the SDE:

$$
\frac{\mathrm{d} H(t)}{H(t)}=\alpha_{H} \mathrm{~d} t+\beta_{H} \mathrm{~d} B(t)
$$

where $B(t)$ is a Brownian motion.

Using Ito Lemma, we get:

$$
\begin{aligned}
\mathrm{d}(H x)= & H \mathrm{~d} x+x \mathrm{~d} H+\mathrm{d} H \mathrm{~d} x \\
= & H(t)\left\{\left[x(t)\left(r-\mu_{Y}+\sigma_{Y}^{2}\right)+\left(\left(\frac{\theta^{N S S}}{\psi}\right)-c(t)\right)\right] \mathrm{d} t-x(t) \sigma_{Y} \mathrm{~d} B(t)\right\} \\
& +x(t) H(t)\left[\alpha_{H} \mathrm{~d} t+\beta_{H} \mathrm{~d} B\right]+H(t)\left[\alpha_{H} \mathrm{~d} t+\beta_{H} \mathrm{~d} B\right] \\
& \times\left\{\left[x(t)\left(r-\mu_{Y}+\sigma_{Y}^{2}\right)+\left(\left(\frac{\theta^{N S S}}{\psi}\right)-c(t)\right)\right] \mathrm{d} t-x(t) \sigma_{Y} \mathrm{~d} B(t)\right\}
\end{aligned}
$$




$$
\begin{aligned}
& =H\left\{\left[x(t)\left(r-\mu_{Y}+\sigma_{Y}^{2}\right)+\left(\left(\frac{\theta^{N S S}}{\psi}\right)-c(t)\right)\right] \mathrm{d} t-x(t) \sigma_{Y} \mathrm{~d} B(t)\right\} \\
& +x H\left[\alpha_{H} \mathrm{~d} t+\beta_{H} \mathrm{~d} B\right]-H x \beta_{H} \sigma_{Y} \mathrm{~d} t
\end{aligned}
$$

Collecting terms, and dropping the dependence on $t$, we get:

$$
\begin{aligned}
\mathrm{d}(H x)= & H\left[x\left(r-\mu_{Y}+\sigma_{Y}^{2}\right)+\left(\left(\frac{\theta^{N S S}}{\psi}\right)-c\right)+x \alpha_{H}-x \beta_{H} \sigma_{Y}\right] \mathrm{d} t \\
& +x H\left(-\sigma_{Y}+\beta_{H}\right) \mathrm{d} B
\end{aligned}
$$

Moving $\left(\left(\frac{\theta^{\text {NSS }}}{\psi}\right)-c\right) \mathrm{d} t$ to the left-hand side, we get:

$$
\begin{aligned}
& \mathrm{d}(H x)-H\left(\left(\frac{\theta^{N S S}}{\psi}\right)-c\right) \mathrm{d} t \\
& =H\left[x\left(r-\mu_{Y}+\sigma_{Y}^{2}\right)+x \alpha_{H}-x \beta_{H} \sigma_{Y}\right] \mathrm{d} t+x H\left(-\sigma_{Y}+\beta_{H}\right) \mathrm{d} B
\end{aligned}
$$

For $H x-H \int\left(\left(\frac{\theta^{N S S}}{\psi}\right)-c\right)$ ds to be a martingale, we need the drift term to be 0.

Thus,

$$
\mathrm{d}(H x)-H\left(\left(\frac{\theta^{N S S}}{\psi}\right)-c\right) \mathrm{d} t=x H\left(-\sigma_{Y}+\beta_{H}\right) \mathrm{d} B
$$

and

$$
0=H\left[x\left(r-\mu_{Y}+\sigma_{Y}^{2}\right)+x \alpha_{H}-x \beta_{H} \sigma_{Y}\right]
$$

This suggests that:

$$
x \beta_{H} \sigma_{Y}=\left[x\left(r-\mu_{Y}+\sigma_{Y}^{2}\right)+x \alpha_{H}\right]=x\left[\left(r-\mu_{Y}+\sigma_{Y}^{2}\right)+\alpha_{H}\right]
$$

Setting $\alpha_{H}=-r$, we get:

$$
\text { i.e., } \beta_{H}=\frac{-\mu_{Y}+\sigma_{Y}^{2}}{\sigma_{Y}}
$$

Thus,

$$
\beta_{H}=\frac{-\mu_{Y}+\sigma_{Y}^{2}}{\sigma_{Y}}, \alpha_{H}=-r
$$

and, $\mathrm{d}(H x)-H\left(\left(\frac{\theta^{\text {NSS }}}{\psi}\right)-c\right) \mathrm{d} t=x H\left(-\sigma_{Y}+\beta_{H}\right) \mathrm{d} B$

where the SDE for $H(t)$ becomes:

$$
\frac{\mathrm{d} H(t)}{H(t)}=-r \mathrm{~d} t-\frac{\mu_{Y}-\sigma_{Y}^{2}}{\sigma_{Y}} \mathrm{~d} B
$$

which has the solution: 


$$
H(t)=\mathrm{e}^{-\int_{0}^{t} \mathrm{rds}} \exp \left\{\frac{-1}{2} \int_{0}^{t}\left[\frac{\left(\mu_{Y}-\sigma_{Y}^{2}\right)}{\sigma_{Y}}\right]^{2} \mathrm{~d} s-\int_{0}^{t} \frac{\left(\mu_{Y}-\sigma_{Y}^{2}\right)}{\sigma_{Y}} \mathrm{~d} B(s)\right\}
$$

Note that $E\{H(t)\}=1$, for all values of $t$.

\section{The new Optimization Problem:}

The new system dynamics become:

$$
\mathrm{d}(H x)-H\left(\left(\frac{\theta^{N S S}}{\psi}\right)-c\right) \mathrm{d} t=x H\left(-\sigma_{Y}+\beta_{H}\right) \mathrm{d} B
$$

Integrating both sides, between 0 and $\tau^{S S}$, we get:

$$
\begin{gathered}
H\left(\tau^{S S}\right) x\left(\tau^{S S}\right)-H(0) x(0)-\int_{0}^{\tau^{S S}} H(s)\left(\left(\frac{\theta^{N S S}}{\psi}\right)-c(s)\right) \mathrm{d} s \\
=\int_{0}^{\tau^{S S}} x(s) H(s)\left(-\sigma_{Y}+\beta_{H}\right) \mathrm{d} B(s) \\
\text { i.e., } \quad H\left(\tau^{S S}\right) x\left(\tau^{S S}\right)-\int_{0}^{\tau^{S S}} H(s)\left(\left(\frac{\theta^{N S S}}{\psi}\right)-c(s)\right) \mathrm{d} s \\
=H(0) x(0)+\int_{0}^{\tau^{S S}} x(s) H(s)\left(-\sigma_{Y}+\beta_{H}\right) \mathrm{d} B(s)
\end{gathered}
$$

Taking the expectation of both sides, we get:

$$
\begin{aligned}
& E\left\{H\left(\tau^{S S}\right) x\left(\tau^{S S}\right)-\int_{0}^{\tau^{S S}} H(s)\left(\left(\frac{\theta^{N S S}}{\psi}\right)-c(s)\right) \mathrm{d} s\right\} \\
& =E\{H(0) x(0)\}=x(0) E\{H(0)\}=x(0)
\end{aligned}
$$

where we used the fact that $E\{H(0)\}=1$.

The optimization problem could now be stated as follows:

Find $c(t)$ that maximizes $V(c(0))$ : (Equation (A.11)) subject to the constraint: (Equation (A.18)) with (Equation (A.10))

Using the Lagrange multiplier method, we need to find:

$$
\begin{aligned}
& \max _{c(s), x\left(\tau^{S S}\right)} E\left\{\int_{0}^{\tau^{S S}} \mathrm{e}^{-\rho s} \frac{c(s)^{1-\gamma}}{1-\gamma} \mathrm{d} s-U_{x}\left(x\left(\tau^{S S}\right)\right)\right. \\
& \left.-\Lambda\left[H\left(\tau^{S S}\right) x\left(\tau^{S S}\right)-\int_{0}^{\tau^{S S}} H(s)\left(\left(\frac{\theta^{N S S}}{\psi}\right)-c(s)\right) \mathrm{d} s-x(0)\right]\right\}
\end{aligned}
$$

which has the form:

$$
\begin{aligned}
& \max _{c(s), x\left(\tau^{s S}\right)} E\left\{\int_{0}^{\tau^{s S}}\left[\mathrm{e}^{-\rho s} \frac{c(s)^{1-\gamma}}{1-\gamma}+\Lambda H(s)\left(\left(\frac{\theta^{N S S}}{\psi}\right)-c(s)\right)\right] \mathrm{d} s\right. \\
& \left.-U_{x}\left(x\left(\tau^{S S}\right)\right)-\Lambda\left[H\left(\tau^{S S}\right) x\left(\tau^{S S}\right)-x(0)\right]\right\}=0
\end{aligned}
$$

where $\Lambda$ is the Lagrange multiplier. Assuming that the conditions for the ex- 
change of the derivative and expectation are satisfied, taking the derivative for $c(t)$, we get:

$$
\begin{aligned}
& \frac{\partial}{\partial c(s)} E\left\{\int_{0}^{\tau^{s S}}\left[\mathrm{e}^{-\rho s} \frac{c(s)^{1-\gamma}}{1-\gamma}+\Lambda H(s)\left(\left(\frac{\theta^{N S S}}{\psi}\right)-c(s)\right)\right] \mathrm{d} s\right\}=0 \\
& \text { i.e., } \frac{\partial}{\partial c(s)}\left[\mathrm{e}^{-\rho s} \frac{c(s)^{1-\gamma}}{1-\gamma}+\Lambda H(s)\left(\left(\frac{\theta^{N S S}}{\psi}\right)-c(s)\right)\right]=0
\end{aligned}
$$

which yields:

$$
\begin{aligned}
& \mathrm{e}^{-\rho s} c(s)^{-\gamma}-\Lambda H(s)=0 \\
& \text { i.e., } c(s)^{-\gamma}=\mathrm{e}^{\rho s} \Lambda H(s)
\end{aligned}
$$

Taking the ln of both sides, we get:

$$
-\gamma \ln c(s)=\rho s+\ln \Lambda H(s)
$$

and

$$
\ln c(s)=-\frac{\rho s}{\gamma}+\left(\frac{-1}{\gamma}\right) \ln \Lambda H(s)
$$

Thus,

$$
c(t)=\mathrm{e}^{-\rho t / \gamma}(\Lambda)^{(-1 / \gamma)}(H(t))^{(-1 / \gamma)}, 0<t \leq \tau^{S S}
$$

Note that $\Lambda$ is a constant deterministic value.

An SDE for $c(t)$ :

We now derive an SDE for the optimal normalized $c(t), 0<t \leq \tau^{S S}$. Since Equation (A.19) then,

$$
\begin{aligned}
\mathrm{d} c(t)= & \mathrm{e}^{-\rho t / \gamma}(\Lambda)^{(-1 / \gamma)} \mathrm{d}(H(t))^{(-1 / \gamma)} \\
& +(-\rho / \gamma) \mathrm{e}^{-\rho t / \gamma}(\Lambda)^{(-1 / \gamma)}(H(t))^{(-1 / \gamma)} \mathrm{d} t, 0<t \leq \tau^{S S}
\end{aligned}
$$

since Equation (A.15).

If we define $y=H^{\alpha}$, using Ito Lemma, we get:

$$
\begin{aligned}
\mathrm{d} y & =\frac{\partial H^{\alpha}}{\partial H} \mathrm{~d} H+\frac{1}{2} \frac{\partial^{2} H^{\alpha}}{\partial H^{2}}(\mathrm{~d} H)^{2} \\
& =\alpha H^{\alpha-1} H\left(-r \mathrm{~d} t-\frac{\left(\mu_{Y}-\sigma_{Y}^{2}\right)}{\sigma_{Y}} \mathrm{~d} B\right)+\frac{1}{2} \alpha(\alpha-1) H^{\alpha-2} H^{2}\left(\frac{\left(\mu_{Y}-\sigma_{Y}^{2}\right)}{\sigma_{Y}}\right)^{2} \mathrm{~d} t \\
& =\alpha H^{\alpha}\left(-r \mathrm{~d} t-\frac{\left(\mu_{Y}-\sigma_{Y}^{2}\right)}{\sigma_{Y}} \mathrm{~d} B\right)+\frac{1}{2} \alpha(\alpha-1) H^{\alpha}\left(\frac{\left(\mu_{Y}-\sigma_{Y}^{2}\right)}{\sigma_{Y}}\right)^{2} \mathrm{~d} t
\end{aligned}
$$

Finally, we get:

$$
\mathrm{d}(H(t))^{\alpha}=-\alpha(H(t))^{\alpha}\left\{\left[r+\frac{(1-\alpha)}{2}\left(\frac{\left(\mu_{Y}-\sigma_{Y}^{2}\right)}{\sigma_{Y}}\right)^{2}\right] \mathrm{d} t+\frac{\left(\mu_{Y}-\sigma_{Y}^{2}\right)}{\sigma_{Y}} \mathrm{~d} B(t)\right\}
$$


Setting $\alpha=(-1 / \gamma)$, we get:

$$
\begin{aligned}
& \mathrm{d}(H(t))^{(-1 / \gamma)}=(1 / \gamma)(H(t))^{(-1 / \gamma)}\left\{\left[r+\frac{(1+1 / \gamma)}{2}\left(\frac{\left(\mu_{Y}-\sigma_{Y}^{2}\right)}{\sigma_{Y}}\right)^{2}\right] \mathrm{d} t+\frac{\left(\mu_{Y}-\sigma_{Y}^{2}\right)}{\sigma_{Y}} \mathrm{~d} B(t)\right\} \\
& \mathrm{d} c(t)=\mathrm{e}^{-\rho t / \gamma}(\Lambda)^{(-1 / \gamma)}(1 / \gamma)(H(t))^{(-1 / \gamma)}\left\{\left[r+\frac{(1+1 / \gamma)}{2}\left(\frac{\left(\mu_{Y}-\sigma_{Y}^{2}\right)}{\sigma_{Y}}\right)^{2}\right] \mathrm{d} t+\frac{\left(\mu_{Y}-\sigma_{Y}^{2}\right)}{\sigma_{Y}} \mathrm{~d} B(t)\right\} \\
& +(-\rho / \gamma) \mathrm{e}^{-\rho t / \gamma}(\Lambda)^{(-1 / \gamma)}(H(t))^{(-1 / \gamma)} \mathrm{d} t, \quad 0<t \leq \tau^{S S}
\end{aligned}
$$

By collecting terms, we get:

$$
\begin{aligned}
& \mathrm{d} c(t)=\mathrm{e}^{-\rho t / \gamma}(\Lambda)^{(-1 / \gamma)}(1 / \gamma)(H(t))^{(-1 / \gamma)}\left\{\left[r+\frac{(1+1 / \gamma)}{2}\left(\frac{\left(\mu_{Y}-\sigma_{Y}^{2}\right)}{\sigma_{Y}}\right)^{2}-\rho\right] \mathrm{d} t+\frac{\left(\mu_{Y}-\sigma_{Y}^{2}\right)}{\sigma_{Y}} \mathrm{~d} B(t)\right\}, 0<t \leq \tau^{S S} \\
& \text { Since } c(t)=\mathrm{e}^{-\rho t / \gamma}(\Lambda)^{(-1 / \gamma)}(H(t))^{(-1 / \gamma)}, 0<t \leq \tau^{S S} \\
& \text { then, } \\
& \mathrm{d} c(t)=c(t)\left(\frac{1}{\gamma}\right)\left\{\left[r+\frac{(1+1 / \gamma)}{2}\left(\frac{\left(\mu_{Y}-\sigma_{Y}^{2}\right)}{\sigma_{Y}}\right)^{2}-\rho\right] \mathrm{d} t+\frac{\left(\mu_{Y}-\sigma_{Y}^{2}\right)}{\sigma_{Y}} \mathrm{~d} B(t)\right\}, 0<t \leq \tau^{S S}
\end{aligned}
$$

Equation (A.23) shows that the optimal normalized consumption, $c(t)$, follows a Geometric Brownian motion, with linear trend coefficient

$$
\begin{aligned}
& \left(\frac{1}{\gamma}\right)\left[r+\frac{(1+1 / \gamma)}{2}\left(\frac{\left(\mu_{Y}-\sigma_{Y}^{2}\right)}{\sigma_{Y}}\right)^{2}-\rho\right] . \text { For the estimated values of Egypt, } \\
& \left(\frac{1}{\gamma}\right)\left[r+\frac{(1+1 / \gamma)}{2}\left(\frac{\left(\mu_{Y}-\sigma_{Y}^{2}\right)}{\sigma_{Y}}\right)^{2}-\rho\right]>0 \text {. The value of } \gamma \text { reflects the level of }
\end{aligned}
$$

conservatism. As $\gamma$ increases, we get conservative results.

The corresponding optimal values of the normalized reserves $x(t)$ are obtained by the substitution of Equation (A.23) into the following equation:

$$
\begin{aligned}
& \qquad \mathrm{d} x(t)=\left[x(t)\left(r-\mu_{Y}+\sigma_{Y}^{2}\right)+\left(\left(\frac{\theta^{N S S}}{\psi}\right)-c(t)\right)\right] \mathrm{d} t-x(t) \sigma_{Y} \mathrm{~d} B(t) \\
& \text { Notice that }\left(\left(\frac{\theta^{N S S}}{\psi}\right)-c(t)\right)>0 \text {, which is a constraint on } c(t) \text {. }
\end{aligned}
$$

